Third, socioeconomic status is a stronger predictor of obesity among normal-weight children than among overweight children. The relative risk of becoming obese among children with lower socioeconomic status as compared with children with higher socioeconomic status was greater for normal-weight kindergartners than for overweight kindergartners.

The patterns of the incidence of obesity during childhood indicate the complexities of the socioeconomic context of this health problem and the reach of the risk of obesity across the socioeconomic spectrum in the United States.

Solveig A. Cunningham, Ph.D.

Michael R. Kramer, Ph.D.

K.M. Venkat Narayan, M.D.

Emory University

Atlanta, GA

sargese@emory.edu

Since publication of their article, the authors report no further potential conflict of interest.

DOI: 10.1056/NEJMc1402397

\title{
A Trial of Mass Isoniazid Preventive Therapy for Tuberculosis Control
}

TO THE EDITOR: In reporting the results of the Thibela TB study, Churchyard and colleagues (Jan. 23 issue) ${ }^{1}$ suggest that continuous isoniazid preventive therapy should be considered for persons at highest risk for tuberculosis (e.g., people with human immunodeficiency virus [HIV] infection), even though several studies in sub-Saharan Africa (including theirs) have shown that the protective effect of isoniazid preventive therapy has limited durability among HIV-infected adults. What their study implies, instead, is a need to discourage the use of isoniazid preventive therapy in areas where there is a high incidence of transmission of tuberculosis and HIV infection and to urgently reassess the data regarding the use of isoniazid preventive therapy in these areas, since isoniazid preventive therapy is increasingly recommended in regions of Africa where there is a high burden of tuberculosis and HIV infection. ${ }^{2}$

In high-incidence areas, $4 \%$ of asymptomatic patients who have HIV infection and smearnegative tuberculosis with normal findings on chest radiography have positive sputum cultures for Mycobacterium tuberculosis, ${ }^{3}$ and up to $30 \%$ of persons with HIV-associated tuberculosis discontinue treatment for tuberculosis, in part because of gaps in the health systems in low-income and middle-income countries. ${ }^{4,5}$ Therefore, continuous isoniazid preventive therapy in areas with a high prevalence of tuberculosis and HIV infection may cause drug pressure, the spread of drugresistant tuberculosis, or both, especially since there are limited methods of diagnosis and treatment for people who have latent infection with drug-resistant strains of $M$. tuberculosis.

Kingsley N. Ukwaja, M.B., B.S.

Federal Teaching Hospital

Abakaliki, Nigeria

ukwajakingsley@yahoo.co.uk

Seye Abimbola, M.D.

University of Sydney

Sydney, NSW, Australia

No potential conflict of interest relevant to this letter was reported.

1. Churchyard GJ, Fielding KL, Lewis JJ, et al. A trial of mass isoniazid preventive therapy for tuberculosis control. N Engl J Med 2014;370:301-10.

2. WHO policy on collaborative TB/HIV activities: guidelines for national programmes and other stakeholders. Geneva: World Health Organization, 2012.

3. Swaminathan S, Paramasivan CN, Kumar SR, Mohan V, Venkatesan P. Unrecognised tuberculosis in HIV-infected patients: sputum culture is a useful tool. Int J Tuberc Lung Dis 2004;8: 896-8.

4. Ifebunandu NA, Ukwaja KN, Obi SN. Treatment outcome of HIV-associated tuberculosis in a resource-poor setting. Trop Doct 2012;42:74-6.

5. Rowe KA, Makhubele B, Hargreaves JR, Porter JD, Hausler HP, Pronyk PM. Adherence to TB preventive therapy for HIVpositive patients in rural South Africa: implications for antiretroviral delivery in resource-poor settings? Int J Tuberc Lung Dis 2005;9:263-9.

DOI: 10.1056/NEJMc1402073

TO THE EDITOR: We question the reported benefit of isoniazid in the post hoc analysis in the Thibela TB study, since patients with tuberculosis who were receiving treatment for tuberculosis or isoniazid preventive therapy at the time of the baseline survey were excluded from the interven- 
tion group. A lack of screening in the "control" group may fully explain the short-term differences in the incidence of tuberculosis between the two groups.

In the corresponding editorial, Rubin ${ }^{1}$ draws the conclusion that persons who are involved in the prevention of tuberculosis need to improve case detection, treatment, and adherence, as well as implement other "technical improvements." It seems that - in an era of effective treatment - those of us in the field have become blind to the prevention benefits of nonmedical interventions (e.g., improvements in ventilation ${ }^{2}$ ). There is a growing consensus that biomedical solutions will be insufficient to tackle the tuberculosis epidemic in low-income and middle-income countries. ${ }^{3}$ Given other negative trials of isoniazid preventive therapy ${ }^{4}$ and enhanced case finding, ${ }^{5}$ a renewed focus on environmental interventions is called for. Or, more controversially, perhaps nontargeted isoniazid preventive therapy should be shelved altogether.

Eugene T. Richardson, M.D.
Stanford University
Stanford, CA
etr@stanford.edu
Robin Wood, D.SC.
Desmond Tutu HIV Centre
Cape Town, South Africa
$\quad$ No potential conflict of interest relevant to this letter was re-
ported.

1. Rubin EJ. Troubles with tuberculosis prevention. N Engl J Med 2014;370:375-6.

2. Richardson ET, Morrow CD, Kalil DB, Bekker L-G, Wood R. Shared air: a renewed focus on ventilation for the prevention of tuberculosis transmission. PLoS One (in press).

3. Hargreaves JR, Boccia D, Evans CA, Adato M, Petticrew M, Porter JDH. The social determinants of tuberculosis: from evidence to action. Am J Public Health 2011;101:654-62.

4. Madhi SA, Nachman S, Violari A, et al. Primary isoniazid prophylaxis against tuberculosis in HIV-exposed children. N Engl J Med 2011;365:21-31.

5. Ayles H, Muyoyeta M, Du Toit E, et al. Effect of household and community interventions on the burden of tuberculosis in southern Africa: the ZAMSTAR community-randomised trial. Lancet 2013;382:1183-94.

DOI: 10.1056/NEJMc1402073

THE AUTHORS REPLY: We agree with Ukwaja and Abimbola that screening to identify people with active tuberculosis and link them to effective treatment is an important part of any isoniazid preventive therapy intervention. We also agree that drug resistance is amplified by weak tuberculosis treatment programs. Studies have not shown that isoniazid preventive therapy promotes isoniazid resistance. ${ }^{1}$ The prevention of tuberculosis, including drug-resistant tuberculosis, requires a comprehensive strategy, of which isoniazid preventive therapy is a key component.

Richardson and Wood suggest that the direct effect of isoniazid preventive therapy in our trial was due to screening. We think this is unlikely. In our analysis of the direct effect of isoniazid, there was a dose-response effect: increasing protection against active tuberculosis with the increasing number of months in which isoniazid was dispensed (see Table S4 in the Supplementary Appendix, available with the full text of our article at NEJM.org). Furthermore, to minimize any screening effect, we excluded patients with tuberculosis who were identified in the first 3 months of follow-up (in both groups), and the protective effect of isoniazid was $49 \%$ as compared with the control cohort (Section S10 in the Supplementary Appendix). Thus, we think that isoniazid preventive therapy has a protective effect that is not attributable to screening alone.

We agree that a "combination prevention" approach is required to improve tuberculosis control, including earlier diagnosis of infectious tuberculosis and a reduction in the number of patients who are initially lost to follow-up. Infection control in health facilities, which includes intensified case finding and administrative measures in addition to improved ventilation, is an important and neglected component of tuberculosis prevention. Improved ventilation alone, however, will not address the problem of reactivation of tuberculosis among the millions of people who have latent tuberculosis infection, particularly those at highest risk, such as persons who are HIV-positive. In areas where it is feasible, tuberculin skin testing can identify people at highest risk for reactivation of tuberculosis who are most likely to benefit from preventive therapy. ${ }^{2}$ In areas in which this testing is not feasible, the lack of a tuberculin skin test result should not be an obstacle to delivering an effective intervention. ${ }^{3}$ Continuous isoniazid preventive therapy provides more protection than a 6-month course of isoniazid therapy in patients at highest risk. ${ }^{4}$

Gavin J. Churchyard, M.B., B.Ch., Ph.D.

Aurum Institute

Johannesburg, South Africa

gchurchyard@auruminstitute.org 
Katherine L. Fielding, Ph.D.

Alison D. Grant, M.B., B.S., Ph.D.

London School of Hygiene and Tropical Medicine London, United Kingdom

Since publication of their article, the authors report no further potential conflict of interest.

1. Balcells ME, Thomas SL, Godfrey-Faussett P, Grant AD. Isoniazid preventive therapy and risk for resistant tuberculosis. Emerg Infect Dis 2006;12:744-51.

2. Akolo C, Adetifa I, Shepperd S, Volmink J. Treatment of la- tent tuberculosis infection in HIV infected persons. Cochrane Database Syst Rev 2010;1:CD000171.

3. Guidelines for intensified tuberculosis case-finding and isoniazid preventive therapy for people living with HIV in resource-constrained settings. Geneva: World Health Organization, 2011.

4. Samandari T, Agizew TB, Nyirenda S, et al. 6-Month versus 36-month isoniazid preventive treatment for tuberculosis in adults with HIV infection in Botswana: a randomised, doubleblind, placebo-controlled trial. Lancet 2011;377:1588-98.

\section{Helicase-Primase Inhibitor Pritelivir for HSV-2 Infection}

TO THE EDITOR: In the context of growing herpes simplex virus (HSV) resistance to licensed antiviral agents targeting HSV DNA polymerase, ${ }^{1}$ especially HSV resistance to acyclovir in immunocompromised patients, the results reported by Wald and colleagues (Jan. 16 issue) ${ }^{2}$ spark great interest. In this trial, the specific helicase-primase inhibitor pritelivir exhibited activity for the treatment of HSV-2 genital infection. To date, four mutations leading to reduced HSV-2 susceptibility to pritelivir have been identified within the UL5 gene, encoding HSV-2 helicase. ${ }^{3}$ We performed a sequence analysis of the full-length UL5 gene from 36 acyclovir-sensitive and 6 acyclovirresistant HSV-2 clinical isolates previously described. ${ }^{4}$ Our results showed a weak variability of HSV-2 helicase, with an interstrain identity of more than $99.7 \%$ at both the nucleotide and ami- no acid levels and no significant difference according to the acyclovir-resistance profile. No preexisting mutation conferring resistance to pritelivir was detected, including in the acyclovirresistant HSV-2 isolates. These data provide some information on the polymorphisms of HSV-2 helicase (Fig. 1). Extended genotypic characterization of the HSV helicase-primase complex is required to facilitate the identification of potential mutations of HSV that may confer resistance to helicase-primase inhibitors.

Sonia Burrel, Pharm.D., Ph.D.

Centre d'Immunologie et des Maladies Infectieuses (CIMI)

Paris, France

sonia.burrel@psl.aphp.fr

\section{Caroline Rouard, Pharm.D.}

Antoine Béclère University Hospital Clamart, France

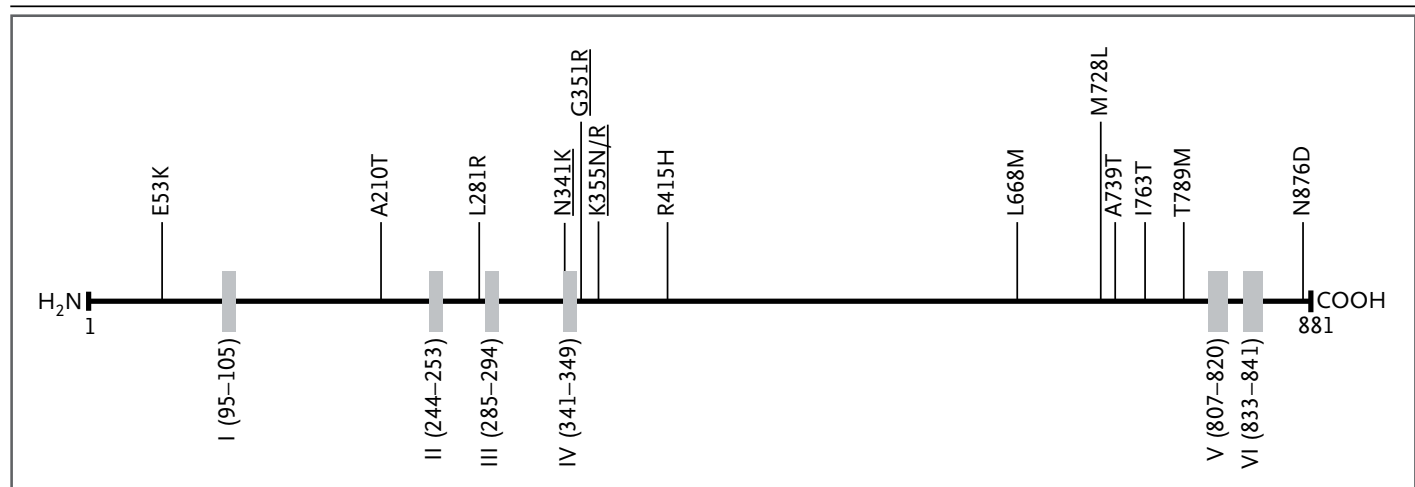

Figure 1. Genetic Variability of HSV-2 Helicase.

Sequencing of HSV-2 helicase ( 881 amino acids) revealed a series of six conserved regions and functional domains ( $\mathrm{l}$ through VI), indicated by the gray boxes. Amino acid changes related to natural polymorphisms are indicated by vertical bars. Underlined mutations are known to be involved in the acquisition of reduced susceptibility to pritelivir. Numbers denote amino acid positions. 